\title{
Pd-mBDPP-Catalyzed Regioselective Internal Arylation of Electron-rich Olefins by Aryl Halides
}

Shifang Liu, ${ }^{a}$ Neil Berry ${ }^{a}$, Nick Thomson, ${ }^{b}$ Alan Pettman, ${ }^{b}$ Zeynab Hyder, ${ }^{a}$ Jun Mo ${ }^{a}$ and Jianliang $\mathrm{Xiao}^{a} *$

\author{
${ }^{a}$ Liverpool Centre for Materials and Catalysis
}

Department of Chemistry, University of Liverpool, Liverpool L69 7ZD, UK

${ }^{\mathrm{b}}$ Pfizer Global Research and Development, Chemical Research and Development Department, Ramsgate Road, Sandwich, Kent CT13 9NJ, UK

\section{Supporting Information}

\section{Contents}

1. Materials and apparatus 2

2. General procedure for the Heck arylation 2

3. Characterization of products 3

4. Methodology of DFT calculations 5

5. Results of DFT calculations $\quad 6$

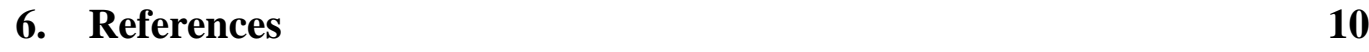

$\begin{array}{ll}\text { 7. Sample NMR spectra } & 11\end{array}$ 


\section{Materials and apparatus}

All chemicals used were purchased from Aldrich or Lancaster and used as received. 1-Butyl-3-methylimidizolium hexafluorophosphate $\left([\mathrm{bmim}]\left[\mathrm{PF}_{6}\right]\right)$ was prepared according to the literature method, ${ }^{1}$ and were dried in vacuo at $70{ }^{\circ} \mathrm{C}$ for $5 \mathrm{~h}$ before use. The reactions were all carried out under a nitrogen atmosphere. The ${ }^{1} \mathrm{H}$ and ${ }^{13} \mathrm{C}$ NMR spectra were recorded on a Gemini 400 spectrometer at $400 \mathrm{MHz}\left({ }^{1} \mathrm{H}\right)$ and $100 \mathrm{MHz}\left({ }^{13} \mathrm{C}\right)$ in ppm with reference to TMS internal standard in $\mathrm{CDCl}_{3}$. Mass spectra were obtained by chemical ionization (CI). All the arylation products were satisfactorily characterized by ${ }^{1} \mathrm{H}$ and ${ }^{13} \mathrm{C}$ NMR, MS, and HRMS and by comparison of their NMR spectra with available literature data. ${ }^{2}$ The ligand meso-2,4-bis(diphenylphosphino)pentane (mBDPP) was synthesized according to a literature procedure. ${ }^{3}$

\section{General procedure for the Heck arylation}

Specific procedures are given for the arylation of 1-bromonaphthalene (1b) by $n$-butyl vinyl ether (2) in DMSO; similar procedures were followed for other coupling reactions. An oven-dried, screw-capped reaction tube containing a stir bar was charged with $\mathbf{1 b}$ (1.0 $\mathrm{mmol}), \mathrm{Pd}(\mathrm{OAc})_{2}(2 \mathrm{~mol} \%), \mathrm{mBDPP}(4 \mathrm{~mol} \%)$, DMSO $(1 \mathrm{~mL})$ under nitrogen at room temperature. Following degassing three times, 2 (2.0 mmol) and $\mathrm{NEt}_{3}(1.5 \mathrm{mmol})$ were injected sequentially under nitrogen. The mixture was heated to $115^{\circ} \mathrm{C}$ and stirred at this temperature for $36 \mathrm{~h}$. The reaction mixture was then cooled to room temperature and acidified by addition of $10 \mathrm{~mL}$ of $20 \% \mathrm{HCl}$. The product was extracted with $\mathrm{CH}_{2} \mathrm{Cl}_{2}(3 \times 10$ $\mathrm{mL})$. The combined organic layer was washed with water until neutrality, dried $\left(\mathrm{MgSO}_{4}\right)$, filtered, and concentrated in vacuo. A small sample was taken for NMR analysis to determine the conversion. The arylated ketone $\mathbf{5 b}$ was isolated in $94 \%$ yield $(160.7 \mathrm{mg}$, 
$0.94 \mathrm{mmol}$ ) as a white powder out of the crude product by flash chromatography on silica gel using a mixture of ethyl acetate and hexane (1:3 to 1:0) as eluant.

\section{Characterization of products}

Characterization of products $5 a-5 m$ : compounds $5 a-5 m$ were characterized by ${ }^{1} \mathrm{H}$ and ${ }^{13} \mathrm{C}$ NMR, MS, and HRMS, and all data were compared with the available data in literature. $^{2}$

2-Acetonaphthone (5a): ${ }^{1} \mathrm{H} \mathrm{NMR}\left(\mathrm{CDCl}_{3}, \delta_{\mathrm{ppm}}\right): 8.46$ (s, $\left.1 \mathrm{H}\right), 8.03$ (dd, $J=8.58,1.75$ $\mathrm{Hz}, 1 \mathrm{H}), 7.95(\mathrm{~d}, J=8.58 \mathrm{~Hz}, 1 \mathrm{H}), 7.88(\mathrm{dd}, J=6.20,5.56 \mathrm{~Hz}, 2 \mathrm{H}), 7.60-7.55(\mathrm{~m}, 2 \mathrm{H})$, $2.71(\mathrm{~s}, 3 \mathrm{H}) .{ }^{13} \mathrm{C} \mathrm{NMR}\left(\mathrm{CDCl}_{3}, \delta_{\mathrm{ppm}}\right): 198.5,136.0,134.9,132.9,130.6,129.9,128.9$, 128.8, 128.2, 127.2, 124.3, 27.1. MS: CI, m/z $188\left[\mathrm{M}+\mathrm{NH}_{4}\right]^{+}(91), 171[\mathrm{M}+\mathrm{H}]^{+}(100), 170$ $\mathrm{M}^{+}$(22), 155 [M-CH$]^{+}$(22); HRMS, Calcd for $\mathrm{C}_{12} \mathrm{H}_{11} \mathrm{O}$ : 171.0810; Found 171.0809.

1-Acetonaphthone (5b) ${ }^{1} \mathrm{H} \mathrm{NMR}\left(\mathrm{CDCl}_{3}, \delta_{\mathrm{ppm}}\right): 8.74(\mathrm{~d}, J=8.75 \mathrm{~Hz}, 1 \mathrm{H}), 7.92(\mathrm{dt}, J$ $=7.84,1.60 \mathrm{~Hz}, 3 \mathrm{H}), 7.60(\mathrm{dt}, J=7.84,1.43 \mathrm{~Hz}, 1 \mathrm{H}), 7.53(\mathrm{~m}, 2 \mathrm{H}), 2.74(\mathrm{~s}, 3 \mathrm{H}) .{ }^{13} \mathrm{C} \mathrm{NMR}$ $\left(\mathrm{CDCl}_{3}, \delta_{\mathrm{ppm}}\right): 202.5,135.9,134.4,133.4,130.6,129.1,128.8,128.5,126.8,126.4,124.7$, 30.4. MS: CI, m/z $188\left[\mathrm{M}+\mathrm{NH}_{4}\right]^{+}(100), 171[\mathrm{M}+\mathrm{H}]^{+}(63), 170 \mathrm{M}^{+}(10), 155\left[\mathrm{M}-\mathrm{CH}_{3}\right]^{+}$ (10); HRMS, Calcd for $\mathrm{C}_{12} \mathrm{H}_{11} \mathrm{O}$ : 171.0810: Found 171.0809.

4'-Methylacetophenone (5c) ${ }^{1} \mathrm{H} \mathrm{NMR}\left(\mathrm{CDCl}_{3}, \delta_{\mathrm{ppm}}\right): 7.86(\mathrm{~d}, J=8.26 \mathrm{~Hz}, 2 \mathrm{H})$, $7.25(\mathrm{~d}, J=8.26 \mathrm{~Hz}, 2 \mathrm{H}), 2.64$ (s, 3H), 2.41 (s, 3H). ${ }^{13} \mathrm{C} \mathrm{NMR}\left(\mathrm{CDCl}_{3}, \delta_{\mathrm{ppm}}\right): 198.3,144.3$, 135.1, 129.6, 128.8, 26.9, 22.0. MS: CI, m/z $152\left[\mathrm{M}+\mathrm{NH}_{4}\right]^{+}(100), 135[\mathrm{M}+\mathrm{H}]^{+}(97), 119$

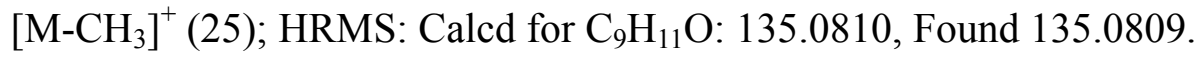

3'-Methylacetophenone (5d) ${ }^{1} \mathrm{H} \mathrm{NMR}\left(\mathrm{CDCl}_{3}, \delta_{\mathrm{ppm}}\right): 7.78(\mathrm{~m}, 2 \mathrm{H}), 7.37(\mathrm{~m}, 2 \mathrm{H})$, $2.60(\mathrm{~s}, 3 \mathrm{H}), 2.42(\mathrm{~s}, 3 \mathrm{H}) .{ }^{13} \mathrm{C} \mathrm{NMR}\left(\mathrm{CDCl}_{3}, \delta_{\mathrm{ppm}}\right): 197.0,136.9,135.7,132.4,127.4,127.0$, 124.2, 25.2, 19.9. MS: CI, m/z $152\left[\mathrm{M}+\mathrm{NH}_{4}\right]^{+}(100), 135[\mathrm{M}+\mathrm{H}]^{+}(30), 134 \mathrm{M}^{+}(<2), 119$ 
$\left[\mathrm{M}-\mathrm{CH}_{3}\right]^{+}(10)$; HRMS, Calcd for $\mathrm{C}_{9} \mathrm{H}_{11} \mathrm{O}$ : 135.0810; Found 135.0808.

Acetophenone (5e) ${ }^{1} \mathrm{H}$ NMR $\left(\mathrm{CDCl}_{3}, \delta_{\mathrm{ppm}}\right): 7.98(\mathrm{~d}, J=7.16 \mathrm{~Hz}, 2 \mathrm{H}), 7.55$ (t, $J=$ $7.16 \mathrm{~Hz}, 1 \mathrm{H}), 7.47$ (t, $J=7.31 \mathrm{~Hz}, 2 \mathrm{H}), 2.62(\mathrm{~s}, 3 \mathrm{H}) .{ }^{13} \mathrm{C} \mathrm{NMR}\left(\mathrm{CDCl}_{3}, \delta_{\mathrm{ppm}}\right): 198.6,137.5$, 133.5, 128.9, 128.7, 27.0. MS: CI, m/z $138\left[\mathrm{M}+\mathrm{NH}_{4}\right]^{+}(100), 121[\mathrm{M}+\mathrm{H}]^{+}(34), 120 \mathrm{M}^{+}(5)$,

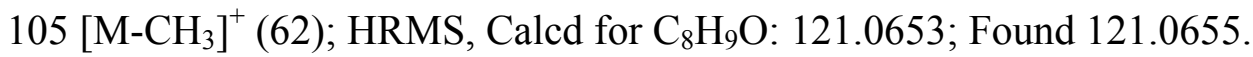

4'-Fluoroacetophenone (5f) ${ }^{1} \mathrm{H} \mathrm{NMR}\left(\mathrm{CDCl}_{3}, \delta_{\mathrm{ppm}}\right): 7.99$ (m, 2H), 7.16 (t, $J=8.68$ $\mathrm{Hz}, 2 \mathrm{H}), 2.60$ (s, 3H). ${ }^{13} \mathrm{C} \mathrm{NMR}\left(\mathrm{CDCl}_{3}, \delta_{\mathrm{ppm}}\right): 197.3,166.6\left(\mathrm{~d}, J_{C F}=254.50 \mathrm{~Hz}\right), 134.4$, $131.9\left(\mathrm{~d}, J_{C F}=9.46 \mathrm{~Hz}\right), 116.5\left(\mathrm{~d}, J_{C F}=22.13 \mathrm{~Hz}\right), 27.3 . \mathrm{MS}: \mathrm{CI}, \mathrm{m} / \mathrm{z} 156\left[\mathrm{M}+\mathrm{NH}_{4}\right]^{+}(100)$, $139[\mathrm{M}+\mathrm{H}]^{+}(10), 138 \mathrm{M}^{+}$(27), $123\left[\mathrm{M}-\mathrm{CH}_{3}\right]^{+}$(78); HRMS, Calcd for $\mathrm{C}_{8} \mathrm{H}_{11} \mathrm{FON}$ : 156.0825, Found 156.0826.

4'-Methoxyacetophenone (5g) ${ }^{1} \mathrm{H} \mathrm{NMR}\left(\mathrm{CDCl}_{3}, \delta_{\mathrm{ppm}}\right): 7.94(\mathrm{~d}, J=8.90 \mathrm{~Hz}, 2 \mathrm{H})$, $6.93(\mathrm{~d}, J=8.90 \mathrm{~Hz}, 2 \mathrm{H}), 3.87$ (s, 3H), $2.56(\mathrm{~s}, 3 \mathrm{H}) .{ }^{13} \mathrm{C} \mathrm{NMR}\left(\mathrm{CDCl}_{3}, \delta_{\mathrm{ppm}}\right): 197.1,163.8$, 130.9, 130.7, 114.0, 55.8, 26.7. MS: CI, m/z $168\left[\mathrm{M}+\mathrm{NH}_{4}\right]^{+}(38), 151[\mathrm{M}+\mathrm{H}]^{+}(100), 135$ $\left[\mathrm{M}-\mathrm{CH}_{3}\right]^{+}$(16); HRMS, Calcd for $\mathrm{C}_{9} \mathrm{H}_{11} \mathrm{O}_{2}$ : 151.0759; Found 151.0755.

3'-Methoxyacetophenone (5h) ${ }^{1} \mathrm{H} \mathrm{NMR}\left(\mathrm{CDCl}_{3}, \delta_{\mathrm{ppm}}\right): 7.53(\mathrm{~d}, J=7.63 \mathrm{~Hz}, 1 \mathrm{H})$, $7.48(\mathrm{dd}, J=3.02,2.54 \mathrm{~Hz}, 1 \mathrm{H}), 7.37$ (t, $J=7.95 \mathrm{~Hz}, 1 \mathrm{H}), 7.12(\mathrm{ddd}, J=8.11,2.70,0.96$ $\mathrm{Hz}, 1 \mathrm{H}), 3.86(\mathrm{~s}, 3 \mathrm{H}), 2.60(\mathrm{~s}, 3 \mathrm{H}) .{ }^{13} \mathrm{C} \mathrm{NMR}\left(\mathrm{CDCl}_{3}, \delta_{\mathrm{ppm}}\right): 198.3,160.1,138.8,129.9$, 121.5, 120.0, 112.7, 55.8, 27.1. MS: CI, m/z $168\left[\mathrm{M}+\mathrm{NH}_{4}\right]^{+}(100), 151[\mathrm{M}+\mathrm{H}]^{+}(35), 150$ $\mathrm{M}^{+}$(11), 135 [M-CH$]^{+}$(8); HRMS, Calcd for $\mathrm{C}_{9} \mathrm{H}_{11} \mathrm{O}_{2}$ : 151.0759; Found 151.0759.

2'-Methoxyacetophenone (5i) ${ }^{1} \mathrm{H} \mathrm{NMR}\left(\mathrm{CDCl}_{3}, \delta_{\mathrm{ppm}}\right): 7.73(\mathrm{dd}, J=7.63,1.91 \mathrm{~Hz}$, 1H), 7.47 (ddd, $J=7.63,1.91,0.80 \mathrm{~Hz}, 1 \mathrm{H}), 6.98$ (q, $J=7.63 \mathrm{~Hz}, 2 \mathrm{H}), 3.91$ (s, 3H), 2.62 (s, $3 \mathrm{H}) .{ }^{13} \mathrm{C} \mathrm{NMR}\left(\mathrm{CDCl}_{3}, \delta_{\mathrm{ppm}}\right): 200.3,159.3,134.1,131.8,120.9,120.5,111.9,55.8,32.2$.

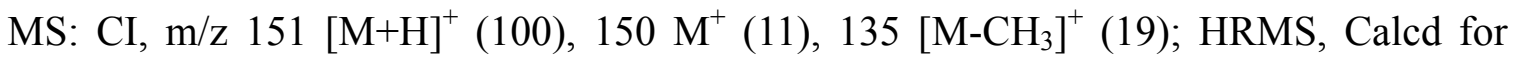
$\mathrm{C}_{9} \mathrm{H}_{11} \mathrm{O}_{2}$ : 151.0759; Found 151.0759. 
4'-Acetylbenzonitrile (5j) ${ }^{1} \mathrm{H} \mathrm{NMR}\left(\mathrm{CDCl}_{3}, \delta_{\mathrm{ppm}}\right): 8.05(\mathrm{~d}, J=8.58 \mathrm{~Hz}, 2 \mathrm{H}), 7.79(\mathrm{~d}$, $J=8.58 \mathrm{~Hz}, 2 \mathrm{H}), 2.66(\mathrm{~s}, 3 \mathrm{H}) .{ }^{13} \mathrm{C} \mathrm{NMR}\left(\mathrm{CDCl}_{3}, \delta_{\mathrm{ppm}}\right): 195.9,140.3,132.9,129.1,118.3$, 116.8, 27.2. MS: CI, m/z $163\left[\mathrm{M}+\mathrm{NH}_{4}\right]^{+}(100), 145 \mathrm{M}^{+}(10), 130\left[\mathrm{M}_{-} \mathrm{CH}_{3}\right]^{+}$(38); HRMS, Calcd for $\mathrm{C}_{9} \mathrm{H}_{11} \mathrm{~N}_{2} \mathrm{O}: 163.0871$, Found 163.0873.

4'-Acetylbenzaldehyde (5k) ${ }^{1} \mathrm{H}$ NMR $\left(\mathrm{CDCl}_{3}, \delta_{\mathrm{ppm}}\right): 10.11$ (s, $\left.1 \mathrm{H}\right), 8.12$ (d, $J=6.60$ $\mathrm{Hz}, 2 \mathrm{H}), 8.01(\mathrm{~d}, J=6.60 \mathrm{~Hz}, 2 \mathrm{H}), 2.66(\mathrm{~s}, 3 \mathrm{H}) .{ }^{13} \mathrm{C} \mathrm{NMR}\left(\mathrm{CDCl}_{3}, \delta_{\mathrm{ppm}}\right): 197.8,191.9$, 141.6, 139.4, 130.2, 129.2, 27.3. MS: CI, m/z $166\left[\mathrm{M}+\mathrm{NH}_{4}\right]^{+}$(30), $148 \mathrm{M}^{+}(100), 133$ $\left[\mathrm{M}-\mathrm{CH}_{3}\right]^{+}(100)$; HRMS, Calcd for $\mathrm{C}_{9} \mathrm{H}_{12} \mathrm{O}_{2} \mathrm{~N}: 166.0868$, Found 166.0861 .

1,4-Diacetylbezene (5I) ${ }^{1} \mathrm{H}$ NMR $\left(\mathrm{CDCl}_{3}, \delta_{\mathrm{ppm}}\right): 8.04$ (s, 4H), 2.65 (s, 6H). ${ }^{13} \mathrm{C}$ NMR $\left(\mathrm{CDCl}_{3}, \delta_{\mathrm{ppm}}\right): 197.8,140.5,128.8,27.2 . \mathrm{MS}: \mathrm{CI}, \mathrm{m} / \mathrm{z} 180\left[\mathrm{M}+\mathrm{NH}_{4}\right]^{+}(100), 163[\mathrm{M}+\mathrm{H}]^{+}$ (5), $162 \mathrm{M}^{+}$(7), $147\left[\mathrm{M}-\mathrm{CH}_{3}\right]^{+}$(21); HRMS, Calcd for $\mathrm{C}_{10} \mathrm{H}_{14} \mathrm{O}_{2} \mathrm{~N}$ : 180.1024; Found 180.1019.

Methyl-4-acetylbenzoate (5m) ${ }^{1} \mathrm{H} \mathrm{NMR}\left(\mathrm{CDCl}_{3}, \delta_{\mathrm{ppm}}\right): 8.13(\mathrm{~d}, J=8.26 \mathrm{~Hz}, 2 \mathrm{H})$, $8.01(\mathrm{~d}, J=8.27 \mathrm{~Hz}, 2 \mathrm{H}), 3.96$ (s, 3H), 2.65 (s, 3H). ${ }^{13} \mathrm{C} \mathrm{NMR}\left(\mathrm{CDCl}_{3}, \delta_{\mathrm{ppm}}\right): 197.9,166.6$, 140.6, 134.2, 130.2, 128.6, 52.8, 27.2. MS: CI, m/z $196\left[\mathrm{M}+\mathrm{NH}_{4}\right]^{+}(100), 179[\mathrm{M}+\mathrm{H}]^{+}(16)$,

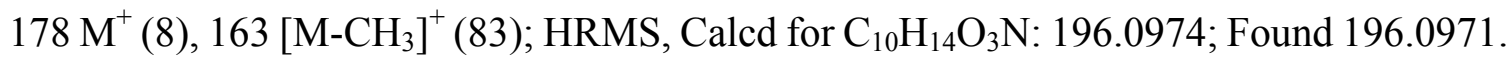

\section{Methodology of DFT calculations}

All calculations were performed on a PC using either Scientific Linux $3.0 .3^{4}$ or Windows $\mathrm{XP}^{5}$ operating systems. All structures were completely optimized at the density functional theory (DFT) level by using the B3LYP functional as implemented in GAMESS $^{6}$ or PCGAMESS. ${ }^{7}$ For all optimizations the $6-31^{*}$ basis set was used for all atoms excluding Pd. Relativistic effects for Pd were addressed by using SBKJC effective core potential (ECP) together with the $\mathrm{SBKJC}^{6}$ basis set. Charges were calculated by 
fitting charges which reproduced the charge, dipole and quadrupole of the molecule. ${ }^{6}$ Calculations were performed in parallel using Linux cluster in the Physics department of Liverpool University. Structures were visualized using Molekel. ${ }^{8}$

\section{Results of DFT calculations}

Optimized geometry of $[\mathrm{PdBr}(\mathrm{Ph})(\mathrm{DPPP})]$ in the singlet ground state in the gas phase

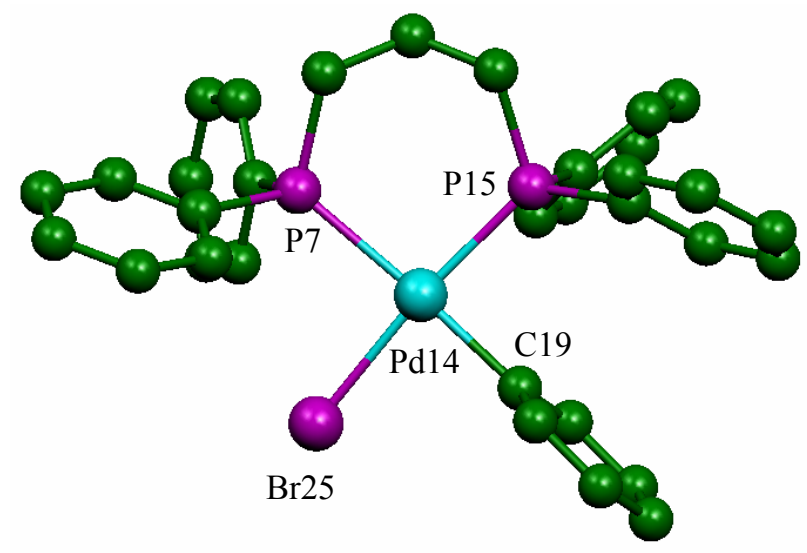

Selected parameters:

\begin{tabular}{|c|c|c|}
\hline \multicolumn{2}{|c|}{ Energy (au) } & -4656.9298691834 \\
\hline \multicolumn{2}{|c|}{ Distance (A) (Pd14-Br25) } & 2.493 \\
\hline \multicolumn{2}{|c|}{ Distance (Å) (P7-Pd14) } & 2.439 \\
\hline \multicolumn{2}{|c|}{ Distance (Å) (P15-Pd14) } & 2.324 \\
\hline \multicolumn{2}{|c|}{ Distance (Å) (Pd14-C19) } & 2.061 \\
\hline \multicolumn{2}{|c|}{ Angle $\left(^{\circ}\right)$ (C19-Pd14-Br25) } & 86.758 \\
\hline \multicolumn{2}{|c|}{ Angle( $\left(^{\circ}\right)$ (Br25-Pd14-P7) } & 90.582 \\
\hline \multicolumn{2}{|c|}{ Angle $\left({ }^{\circ}\right)$ (C19-Pd14-P15) } & 87.516 \\
\hline \multicolumn{2}{|c|}{ Angle( ${ }^{\circ}$ ) (P7-Pd14-P15) } & 95.273 \\
\hline \multirow{5}{*}{ Charge } & P7 & 0.1222 \\
\hline & P15 & 0.3557 \\
\hline & Pd14 & -0.3066 \\
\hline & C19 & 0.1429 \\
\hline & Br25 & -0.3468 \\
\hline
\end{tabular}


Coordinates of $[\mathrm{PdBr}(\mathrm{Ph})(\mathrm{DPPP})]$ in xyz format in Angstroms

68

0.0000000

\begin{tabular}{|c|c|c|c|}
\hline C & -3.03029 & 2.11277 & -1.34979 \\
\hline C & -3.33347 & 0.75754 & -1.15071 \\
\hline C & -4.65939 & ๑. 32281 & -1.29049 \\
\hline C & -5.66432 & 1.22809 & -1.63462 \\
\hline C & -5.35383 & 2.57370 & -1.84217 \\
\hline C & -4.03645 & 3.01366 & -1.69995 \\
\hline $\mathrm{P}$ & -1.95759 & -0.40768 & -0.77748 \\
\hline C & -2.72808 & -1.77052 & ๑.20247 \\
\hline C & -3.10698 & -3.00520 & -0.34844 \\
\hline C & -3.67423 & -3.99877 & 0.45378 \\
\hline C & -3.87324 & -3.77141 & 1.81546 \\
\hline C & -3.49861 & -2.54656 & 2.37331 \\
\hline C & -2.92464 & -1.55418 & 1.57934 \\
\hline $\mathrm{Pd}$ & ๑. 02368 & $\odot .56920$ & 0.25718 \\
\hline$P$ & 1.54668 & $-\odot .61279$ & -1.03982 \\
\hline C & $\odot .90946$ & -1.29118 & -2.66359 \\
\hline C & -0.42887 & -2.04983 & -2.59340 \\
\hline C & -1.66882 & -1.14382 & -2.4670 \\
\hline C & 1.63627 & 1.48251 & 1.15843 \\
\hline C & 2.35978 & 0.88558 & 2.19829 \\
\hline C & 3.42656 & 1.55941 & 2.80569 \\
\hline C & 3.78415 & 2.83933 & 2.38083 \\
\hline C & 3.06192 & 3.44488 & 1.3499 \\
\hline C & 1.99618 & 2.77231 & 0.7446 \\
\hline $\mathrm{Br}$ & -1.40220 & 1.77634 & 1.90755 \\
\hline C & 3.10305 & ๑. 22223 & -1.56568 \\
\hline C & 4.27379 & 0.11853 & $-\odot .79860$ \\
\hline C & 5.42650 & $\odot .81165$ & -1.1686 \\
\hline C & 5.42829 & 1.62127 & -2.3052 \\
\hline C & 4.26504 & 1.74379 & -3.06641 \\
\hline C & 3.10985 & 1.05434 & -2.69702 \\
\hline C & 2.08431 & -2.15469 & -0.18201 \\
\hline C & 1.45521 & -2.52566 & 1.01570 \\
\hline C & 1.80011 & -3.71793 & 1.6571 \\
\hline C & 2.77888 & -4.54831 & 1.1112 \\
\hline C & 3.41395 & -4.18635 & -0.08035 \\
\hline C & 3.06851 & -2.99889 & -0.72428 \\
\hline $\mathrm{H}$ & 1.43053 & 3.27107 & -0.03937 \\
\hline $\mathrm{H}$ & 3.32209 & 4.44842 & 1.0183 \\
\hline $\mathrm{H}$ & 4.61120 & 3.36421 & 2.8535 \\
\hline $\mathrm{H}$ & 3.97234 & 1.08073 & 3.6170 \\
\hline $\mathrm{H}$ & 2.09388 & -0.10669 & 2.55437 \\
\hline $\mathrm{H}$ & 2.21188 & 1.18374 & -3.29455 \\
\hline $\mathrm{H}$ & 4.25197 & 2.38014 & -3.9475 \\
\hline $\mathrm{H}$ & 6.32740 & 2.16090 & -2.5910 \\
\hline $\mathrm{H}$ & 6.32236 & 0.72234 & -0.55994 \\
\hline $\mathrm{H}$ & 4.28318 & -0.49176 & ๑. 0979 \\
\hline $\mathrm{H}$ & $\odot .69672$ & -1.87441 & 1.44367 \\
\hline $\mathrm{H}$ & 1.30375 & -3.99190 & 2.5841 \\
\hline $\mathrm{H}$ & 3.05004 & -5.47430 & 1.61180 \\
\hline $\mathrm{H}$ & 4.18017 & -4.82843 & -0.50733 \\
\hline $\mathrm{H}$ & 3.57929 & -2.72296 & -1.64342 \\
\hline $\mathrm{H}$ & 1.68946 & -1.94892 & -3.06452 \\
\hline $\mathrm{H}$ & 0.81179 & -0.45333 & -3.36349 \\
\hline
\end{tabular}




$\begin{array}{llll}\mathrm{H} & -0.40441 & -2.79138 & -1.78491 \\ \mathrm{H} & -0.52543 & -2.62511 & -3.52382 \\ \mathrm{H} & -2.56993 & -1.67625 & -2.79277 \\ \mathrm{H} & -1.57032 & -0.28485 & -3.14313 \\ \mathrm{H} & -2.96638 & -3.20988 & -1.40515 \\ \mathrm{H} & -3.95966 & -4.94934 & 0.00986 \\ \mathrm{H} & -4.31579 & -4.54446 & 2.43873 \\ \mathrm{H} & -3.64837 & -2.35995 & 3.43363 \\ \mathrm{H} & -2.62832 & -0.60411 & 2.01771 \\ \mathrm{H} & -4.91165 & -0.72001 & -1.11746 \\ \mathrm{H} & -6.69028 & 0.88253 & -1.73441 \\ \mathrm{H} & -6.13849 & 3.27889 & -2.10465 \\ \mathrm{H} & -3.79215 & 4.06276 & -1.84483 \\ \mathrm{H} & -2.01177 & 2.46337 & -1.21051\end{array}$

Optimized geometry of $[\mathrm{PdBr}(\mathrm{Ph})(\mathrm{mBDPP})]$ in the singlet ground state in the gas phase

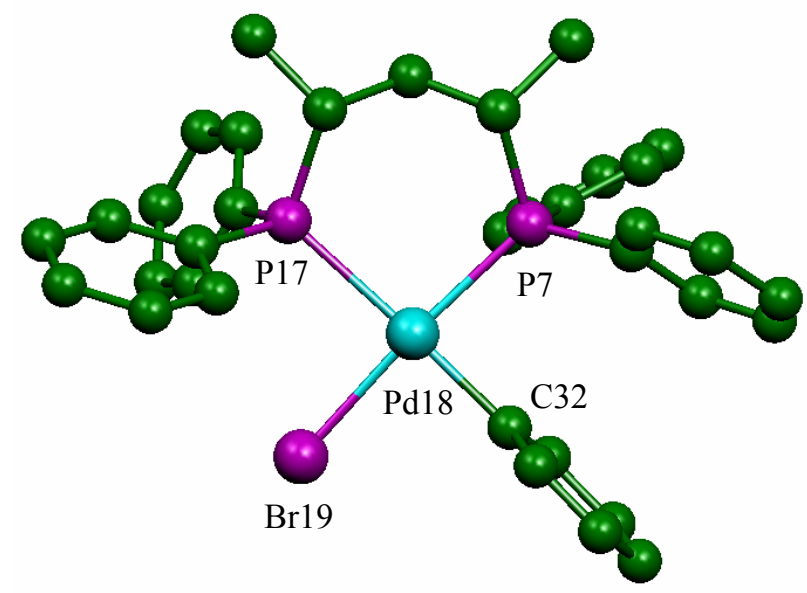

Selected parameters:

\begin{tabular}{|c|c|c|}
\hline \multicolumn{2}{|c|}{ Energy (au) } & -4723.6778492809 \\
\hline \multicolumn{2}{|c|}{ Distance (Å) (Pd18-Br19) } & 2.508 \\
\hline \multicolumn{2}{|c|}{ Distance (Å) (P7-Pd18) } & 2.421 \\
\hline \multicolumn{2}{|c|}{ Distance (Å) (P17-Pd18) } & 2.541 \\
\hline \multicolumn{2}{|c|}{ Distance (A) (Pd18-C32) } & 2.033 \\
\hline \multicolumn{2}{|c|}{ Angle $\left(^{\circ}\right)(\mathrm{C} 32-P d 18-\mathrm{Br} 19)$} & 88.081 \\
\hline \multicolumn{2}{|c|}{ Angle ( $\left.{ }^{\circ}\right)($ Br19-Pd18- P17) } & 90.152 \\
\hline \multicolumn{2}{|c|}{ Angle $\left({ }^{\circ}\right)($ C32-Pd18-P7) } & 87.398 \\
\hline \multicolumn{2}{|c|}{ Angle( $\left.{ }^{\circ}\right)($ 17-Pd18-P7) } & 94.411 \\
\hline \multirow{5}{*}{ Charge } & P7 & 0.0453 \\
\hline & P17 & -0.2355 \\
\hline & Pd18 & -0.0155 \\
\hline & C32 & 0.1034 \\
\hline & Br19 & -0.4859 \\
\hline
\end{tabular}


Coordinates of $[\mathrm{PdBr}(\mathrm{Ph})(\mathrm{mBDPP})]$ in xyz format in Angstroms

74 0.0000000

\begin{tabular}{|c|c|c|c|}
\hline C & 3. 26131 & -2.93578 & -0.56020 \\
\hline C & 2.13282 & -2.19595 & $-\odot .20017$ \\
\hline C & 1.35019 & -2.65357 & 0.85747 \\
\hline C & 1.67447 & -3.82389 & 1.52796 \\
\hline C & 2.79301 & -4.54691 & 1.15690 \\
\hline C & 3.58886 & -4.09845 & 0.11272 \\
\hline$P$ & 1.60145 & -0.67569 & -1.08675 \\
\hline C & 3.13452 & 0.21906 & -1.56656 \\
\hline C & 4.27033 & 0.18849 & -0.76233 \\
\hline C & 5.39121 & 0.93083 & -1.09394 \\
\hline C & 5.39463 & 1.72352 & -2.2282 \\
\hline C & 4.26430 & 1.77953 & -3.0259 \\
\hline C & 3.14353 & 1.03788 & -2.69472 \\
\hline C & 0.92815 & -1.29267 & -2.72553 \\
\hline C & -0.43079 & -2.01649 & -2.59181 \\
\hline C & -1.68183 & -1.10593 & -2.53388 \\
\hline $\mathrm{P}$ & -2.02339 & $-\odot .44 \odot 42$ & -0.8234 \\
\hline $\mathrm{Pd}$ & 0.03361 & 0.57156 & 0.27253 \\
\hline $\mathrm{Br}$ & -1.43075 & 1.84117 & 1.86407 \\
\hline C & -3.38666 & 0.74886 & -1.12752 \\
\hline C & -3.05704 & 2.07495 & -1.3943 \\
\hline C & -4.04429 & 3.00282 & -1.6795 \\
\hline C & -5.37509 & 2.62061 & -1.68423 \\
\hline C & -5.71673 & 1.30767 & -1.40317 \\
\hline C & -4.72886 & 0.37753 & -1.12677 \\
\hline C & -2.75731 & -1.77227 & 0.21674 \\
\hline C & -2.78905 & -3.11943 & -0.1327 \\
\hline C & -3.30559 & -4.06905 & 0.7368 \\
\hline C & -3.79781 & -3.68645 & 1.97102 \\
\hline C & -3.76067 & -2.34973 & 2.33783 \\
\hline C & -3.24109 & -1.40147 & 1.47522 \\
\hline C & 1.64089 & 1.43414 & 1.17055 \\
\hline C & 2.32750 & ๑. 82321 & 2.2157 \\
\hline C & 3.39988 & 1.45040 & 2.8383 \\
\hline C & 3.80836 & 2.70691 & 2.42744 \\
\hline C & 3.12640 & 3.33422 & 1.39556 \\
\hline C & 2.05659 & 2.70581 & 0.77904 \\
\hline $\mathrm{H}$ & 1.52939 & 3.22579 & -0.00261 \\
\hline $\mathrm{H}$ & 3.42335 & 4. 31871 & 1.0756 \\
\hline $\mathrm{H}$ & 4.63657 & 3.19646 & 2.90983 \\
\hline $\mathrm{H}$ & 3.90867 & $\odot .95656$ & 3.64932 \\
\hline $\mathrm{H}$ & 2.02403 & -0.14764 & 2.56805 \\
\hline $\mathrm{H}$ & 2.27743 & 1.11460 & -3.32531 \\
\hline $\mathrm{H}$ & 4.24991 & 2.40194 & -3.90262 \\
\hline $\mathrm{H}$ & 6.26507 & 2.30115 & -2.48260 \\
\hline $\mathrm{H}$ & 6.25501 & 0.89652 & -0.45529 \\
\hline $\mathrm{H}$ & 4.28039 & -0.39366 & 0.13766 \\
\hline $\mathrm{H}$ & 0.48942 & -2.09438 & 1.17400 \\
\hline $\mathrm{H}$ & 1.05663 & -4.15714 & 2.34179 \\
\hline $\mathrm{H}$ & 3.05025 & -5.45041 & 1.68009 \\
\hline $\mathrm{H}$ & 4.46422 & -4.65223 & -0.17556 \\
\hline $\mathrm{H}$ & 3.89574 & -2.59981 & -1.35793 \\
\hline C & 1.90557 & -2.17333 & -3.5186 \\
\hline
\end{tabular}




$\begin{array}{lrrr}\mathrm{H} & 0.75858 & -0.38975 & -3.30300 \\ \mathrm{H} & -0.39846 & -2.70875 & -1.75702 \\ \mathrm{H} & -0.53520 & -2.63890 & -3.47522 \\ \mathrm{C} & -2.86706 & -1.71994 & -3.29595 \\ \mathrm{H} & -1.43463 & -0.18767 & -3.06207 \\ \mathrm{H} & -2.41167 & -3.45186 & -1.07841 \\ \mathrm{H} & -3.32253 & -5.10343 & 0.44298 \\ \mathrm{H} & -4.20158 & -4.42162 & 2.64409 \\ \mathrm{H} & -4.12698 & -2.04191 & 3.30045 \\ \mathrm{H} & -3.18822 & -0.37587 & 1.78716 \\ \mathrm{H} & -5.00930 & -0.63487 & -0.90255 \\ \mathrm{H} & -6.74934 & 1.00770 & -1.39427 \\ \mathrm{H} & -6.14286 & 3.34374 & -1.89432 \\ \mathrm{H} & -3.77392 & 4.02453 & -1.87583 \\ \mathrm{H} & -2.03253 & 2.39436 & -1.35078 \\ \mathrm{H} & 1.51760 & -2.32610 & -4.52142 \\ \mathrm{H} & 2.88544 & -1.72262 & -3.61128 \\ \mathrm{H} & 2.01939 & -3.14782 & -3.05903 \\ \mathrm{H} & -2.58329 & -1.87252 & -4.33354 \\ \mathrm{H} & -3.17424 & -2.67883 & -2.89946 \\ \mathrm{H} & -3.72655 & -1.06330 & -3.28612\end{array}$

\section{References}

(1) Fuller, J.; Carlin, R. T.; de Long, H. C.; Haworth, D. J. Chem. Soc., Chem. Commun. 1994, 299.

(2) Mo, J.; Xu, L.; Xiao, J. J. Am. Chem. Soc. 2005, 127, 751.

(3) Bakos, J.; Tóth, I.; Hell, B.; Markó, L. J. Organomet. Chem. 1985, 279, 23.

(4) https://www.scientificlinux.org/.

(5) http://www.windows.com.

(6) Schmidt, M. W.; Baldridge, K. K.; Boatz, J. A.; Elbert, S. T.; Gordon, M. S.; Jensen, J. H.; Koseki, S.; Matsunaga, N.; Nguyen, K. A.; Su, S. J.; Windus, T. L.; Dupuis, M.; Montgomery, J. A. J. Comput. Chem. 1993, 14, 1347.

(7) Granovsky, A. A. www http://classic.chem.msu.su/gran/gamess/index.html.

(8) Portmann, S.; Lüthi, H. P. CHIMIA, 2000, 54, 766. 


\section{Sample NMR spectra}<smiles>CC(=O)c1ccc2ccccc2c1</smiles>

${ }^{1} \mathrm{H}$ NMR

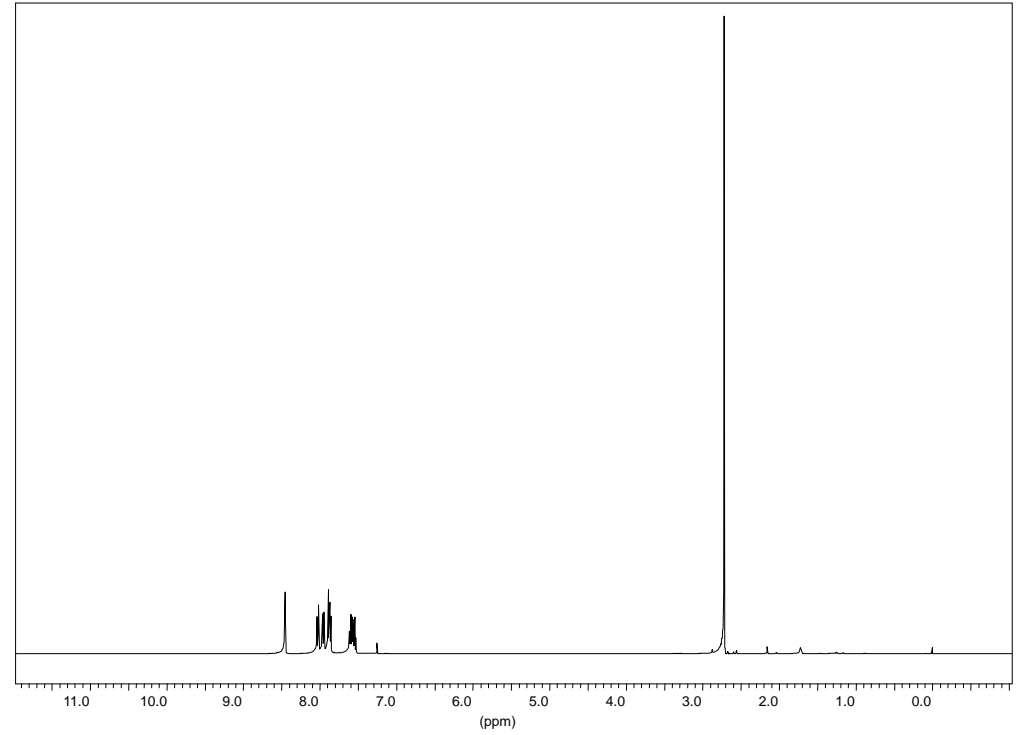

${ }^{13} \mathrm{CNMR}$

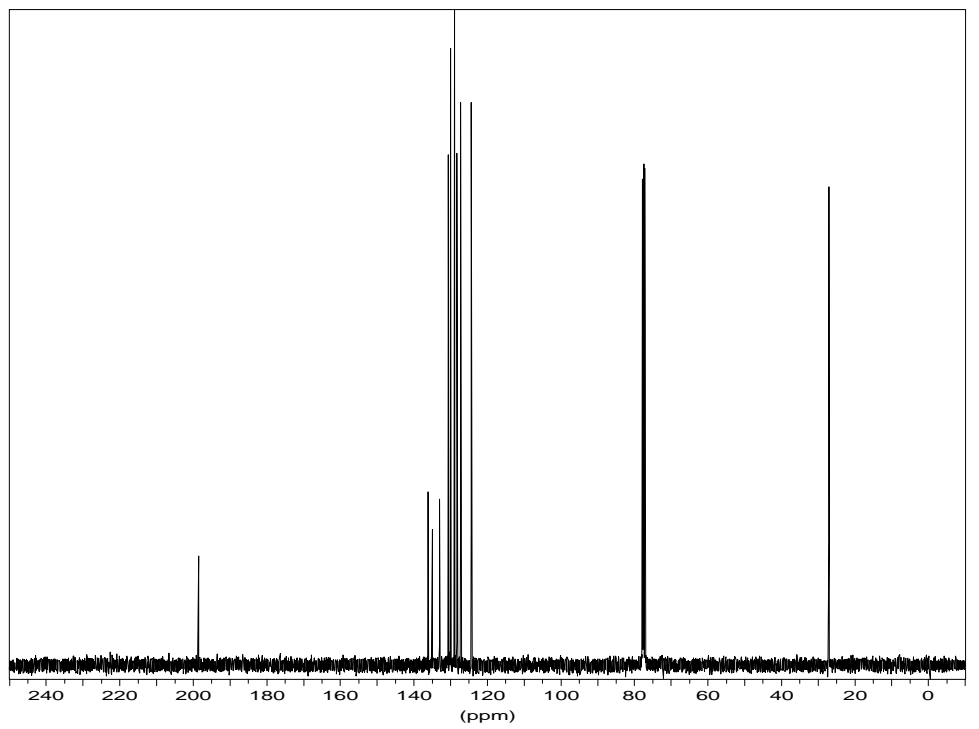


<smiles>CC(=O)c1cccc2ccccc12</smiles>

$5 b$

${ }^{1}$ HNMR

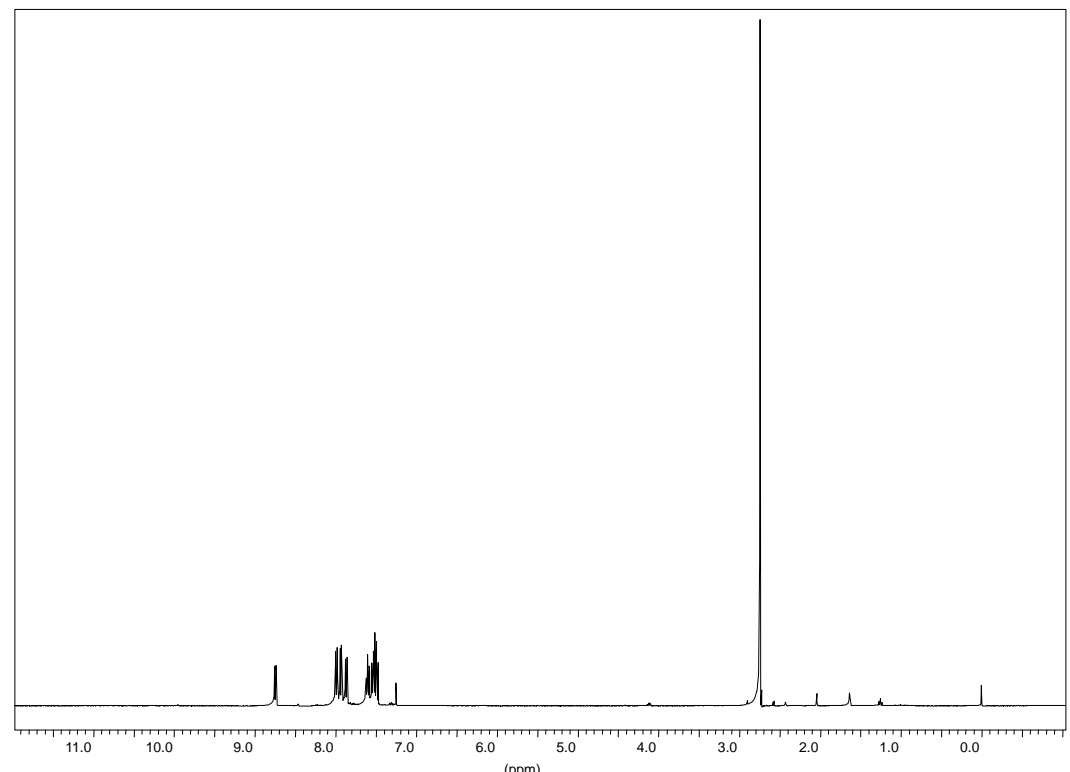

${ }^{13}$ C NMR 5b

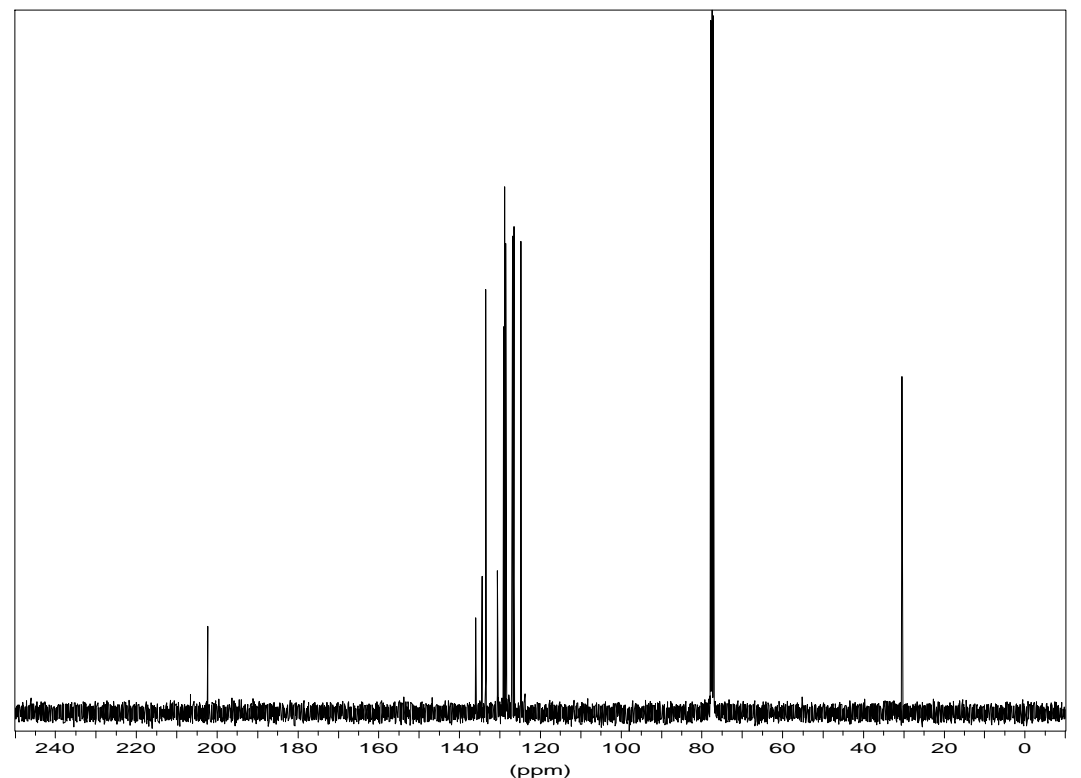


<smiles>CC(=O)c1ccc(C)cc1</smiles>

${ }^{1} \mathrm{H}$ NMR

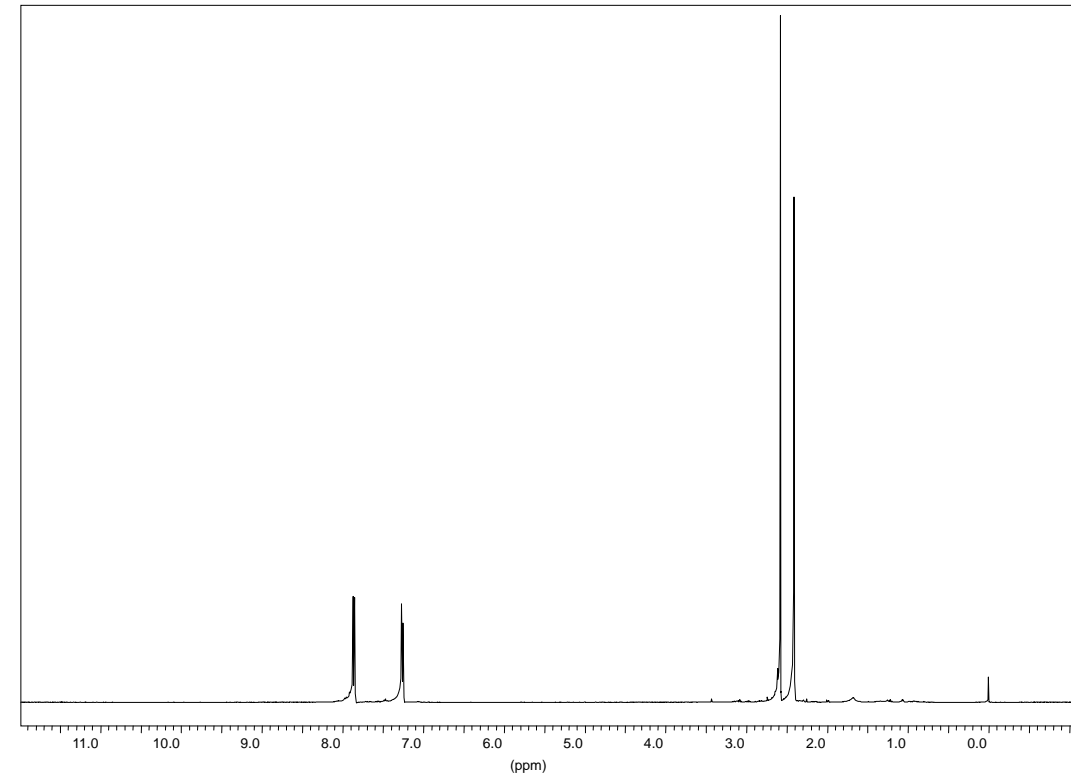

${ }^{13}$ C NMR 5c

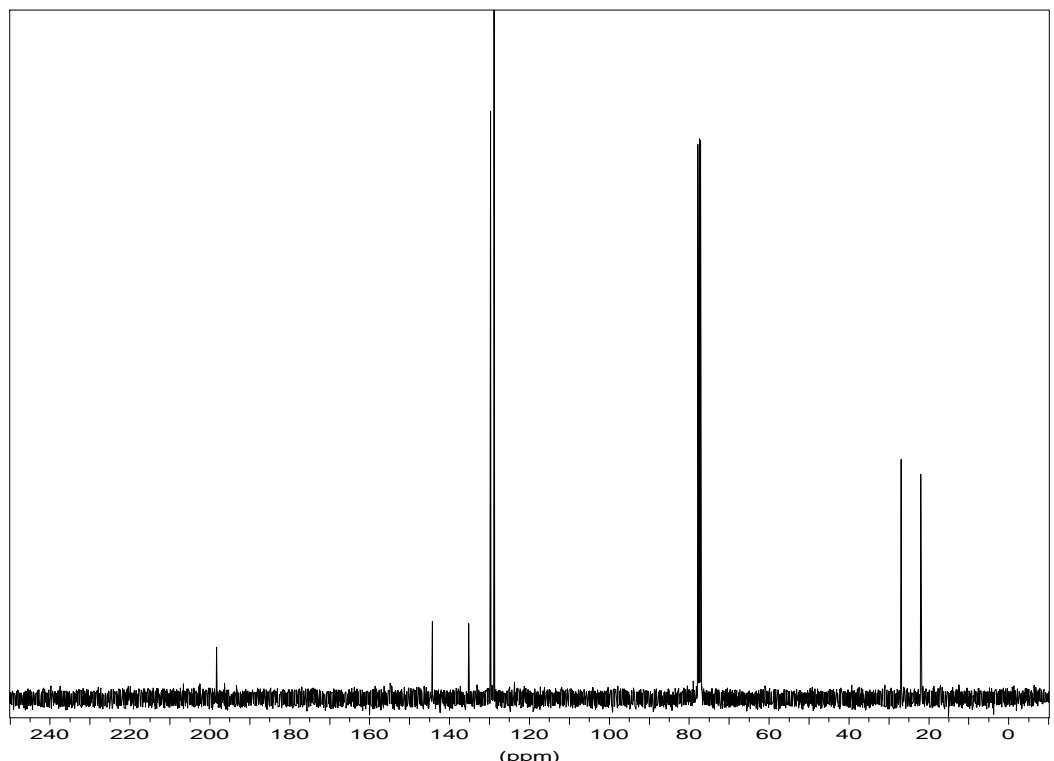




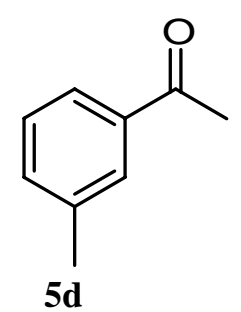

${ }^{1}$ HNMR

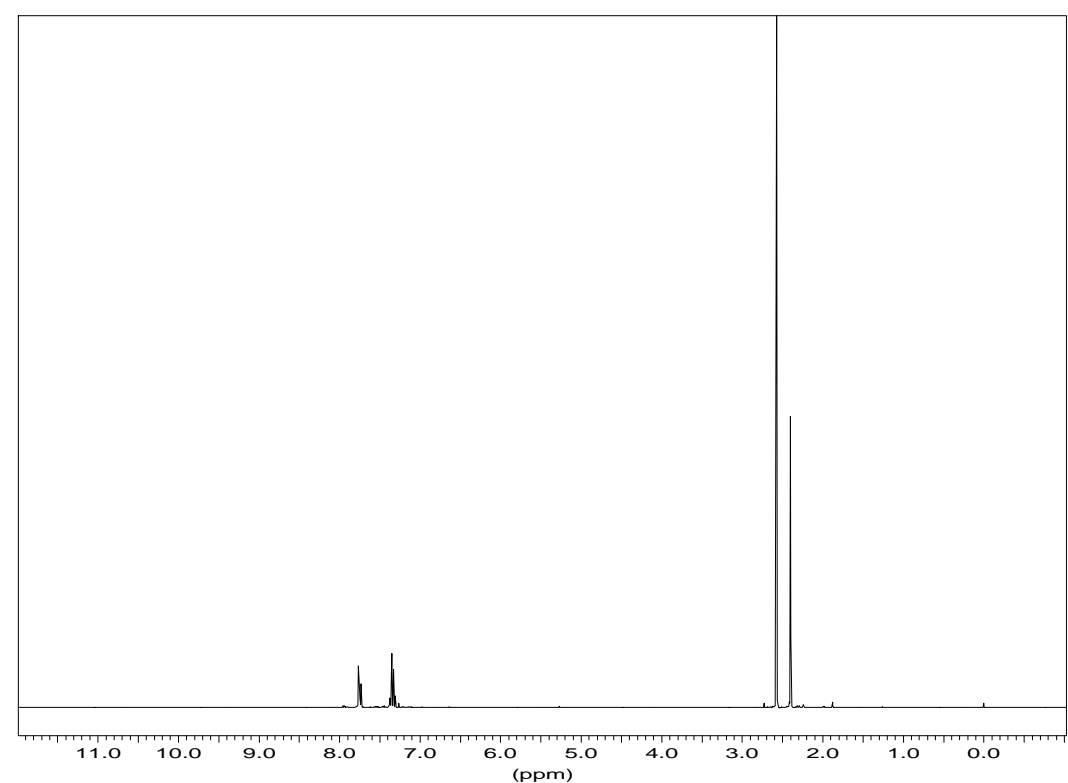

${ }^{13} \mathrm{C}$ NMR

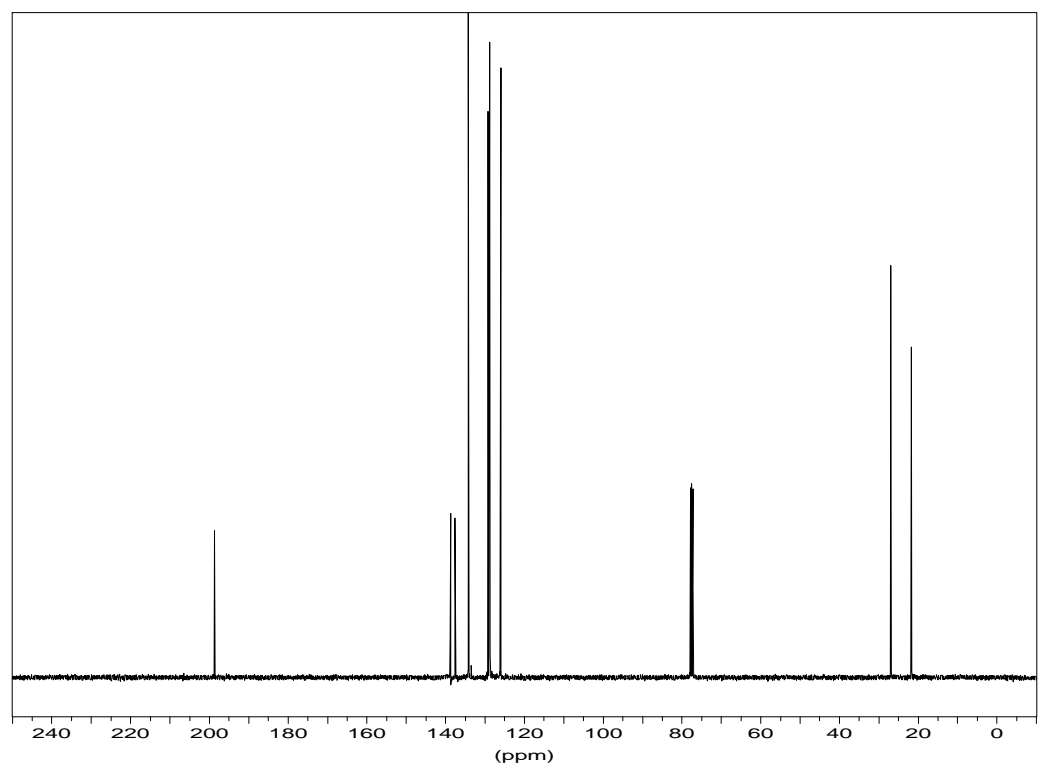




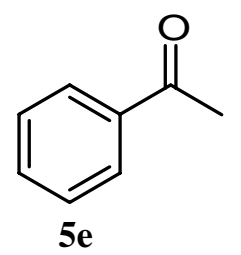

${ }^{1}$ H NMR

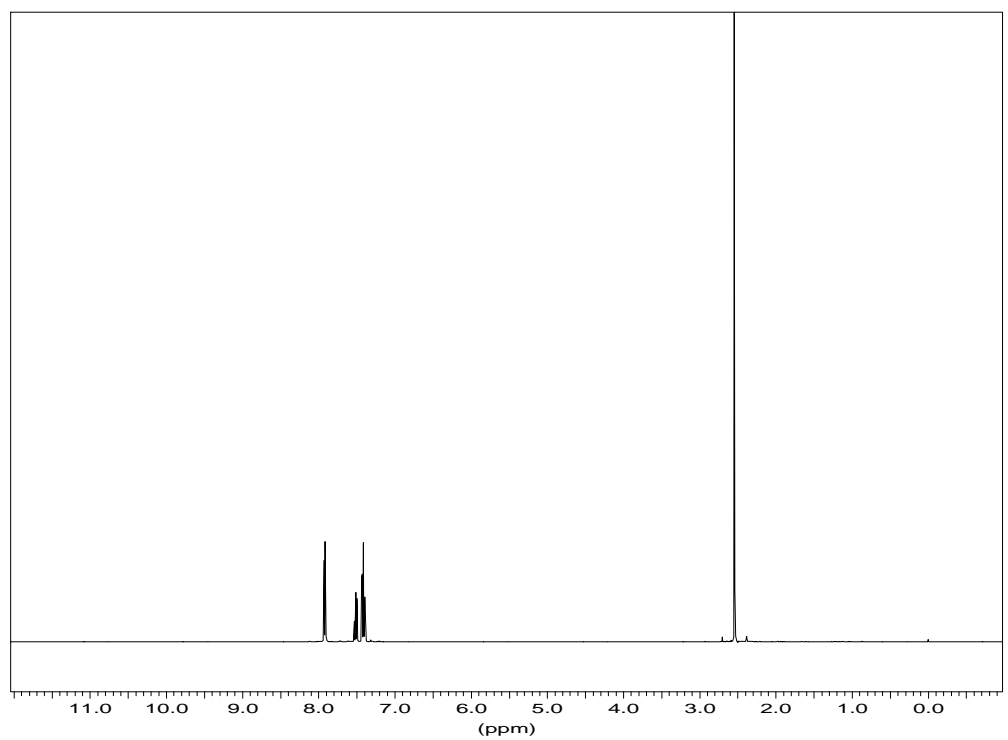

${ }^{13} \mathrm{C}$ NMR

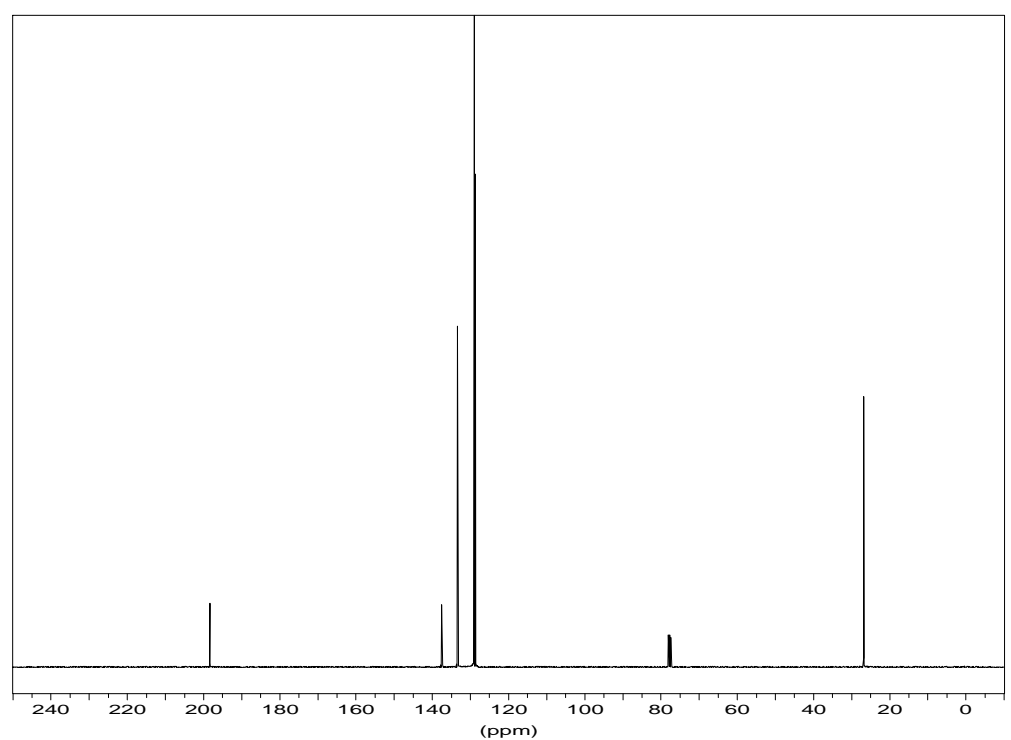


<smiles>CC(=O)c1ccc(F)cc1</smiles>

${ }^{1} \mathrm{H}$ NMR

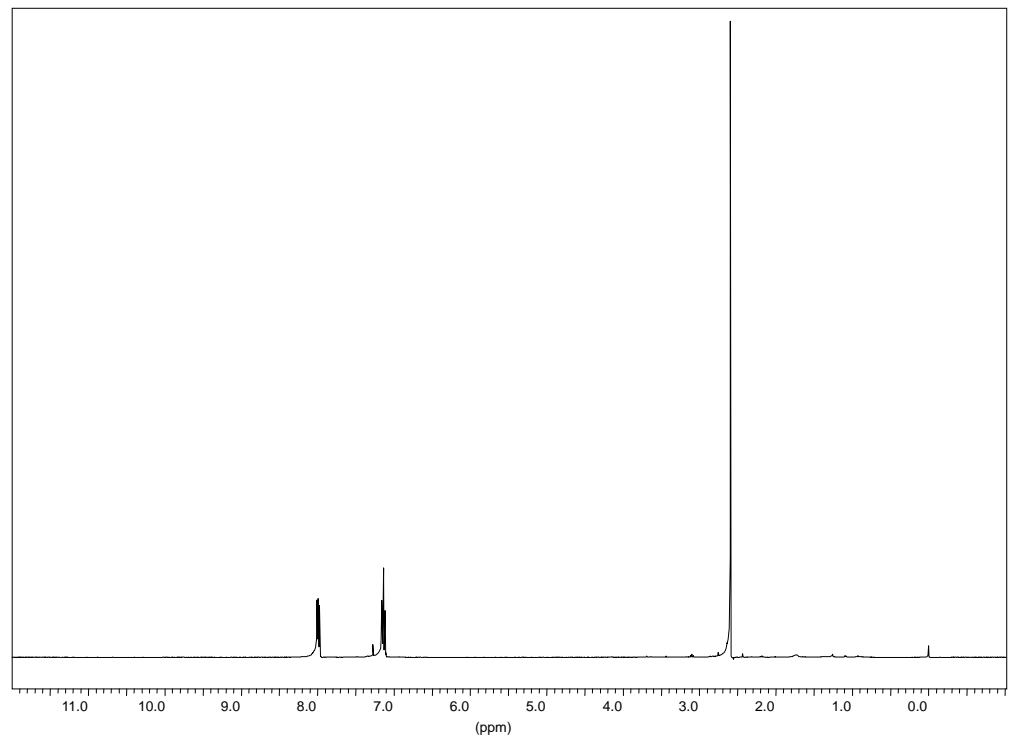

${ }^{13}$ C NMR

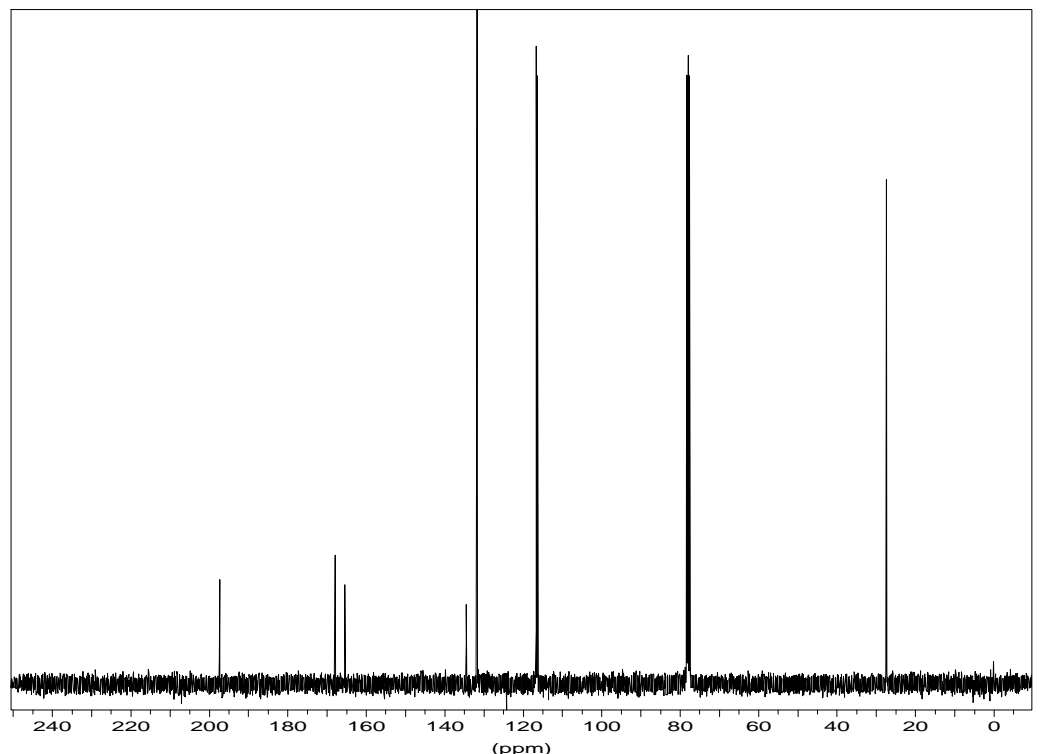


<smiles>COc1ccc(C(C)=O)cc1</smiles>

$5 g$

${ }^{1}$ H NMR

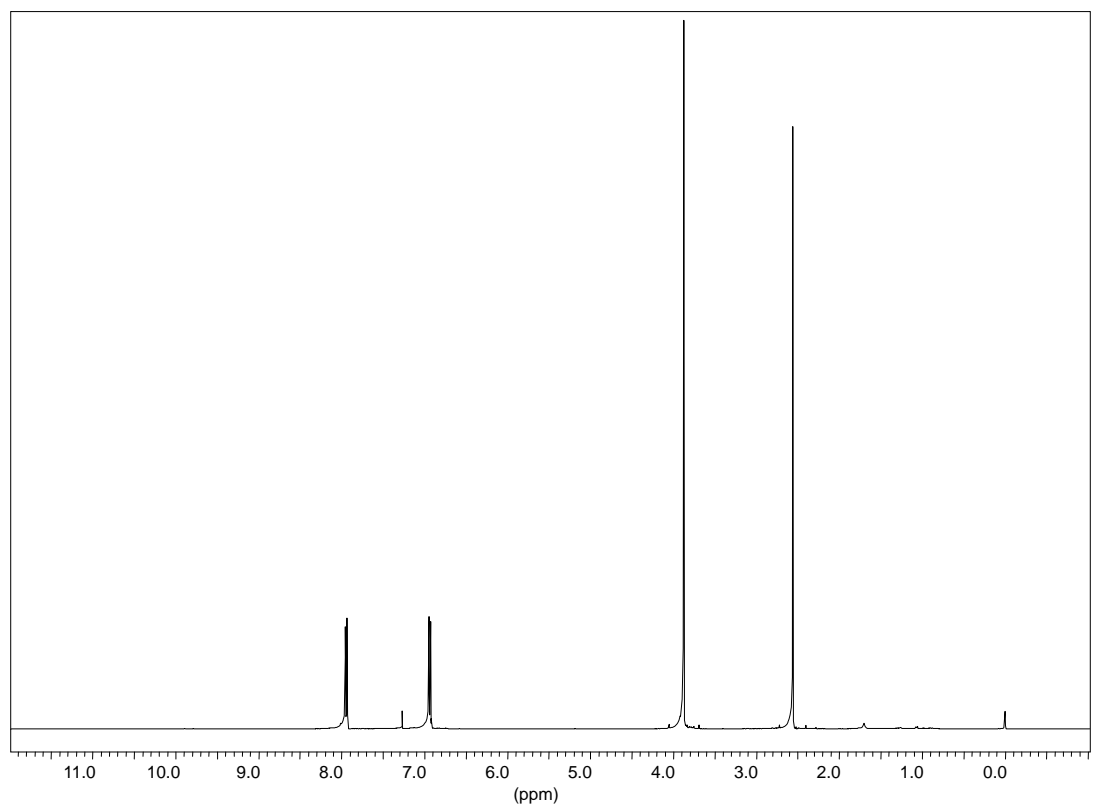

${ }^{13}$ C NMR

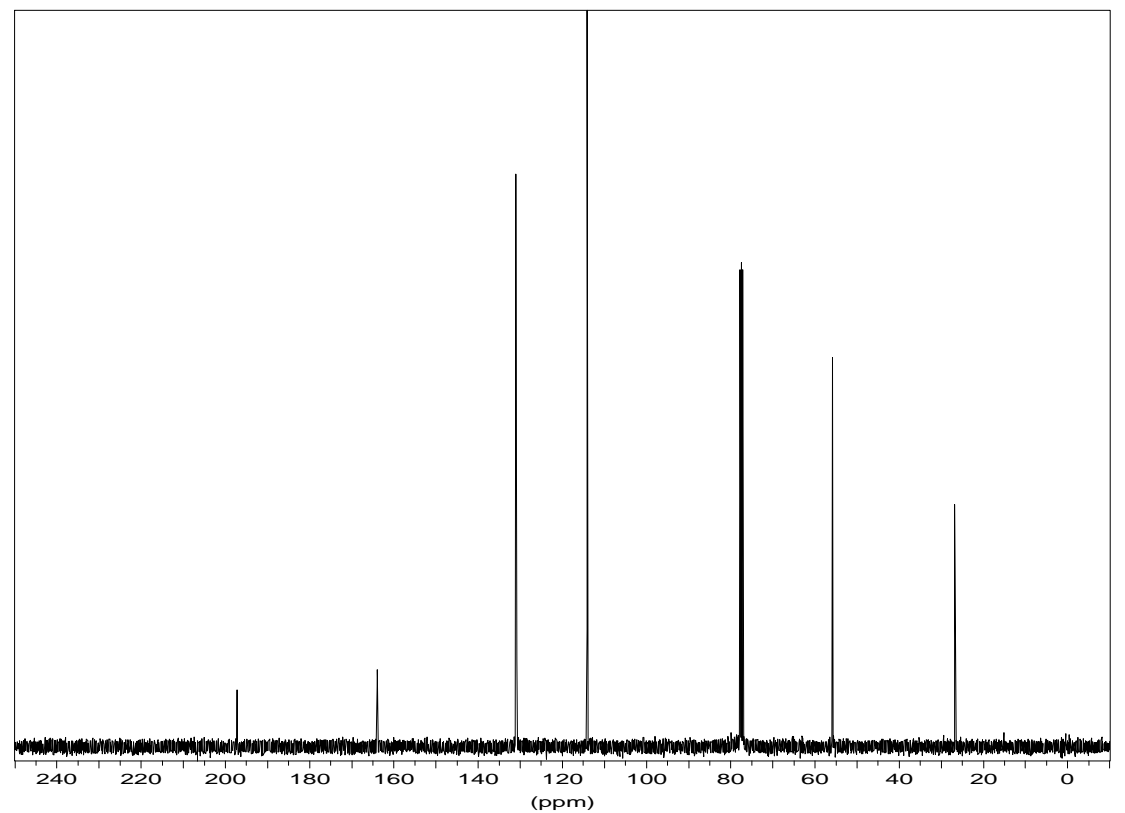




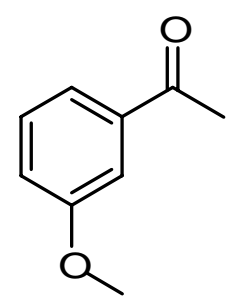

$5 h$

${ }^{1}$ H NMR

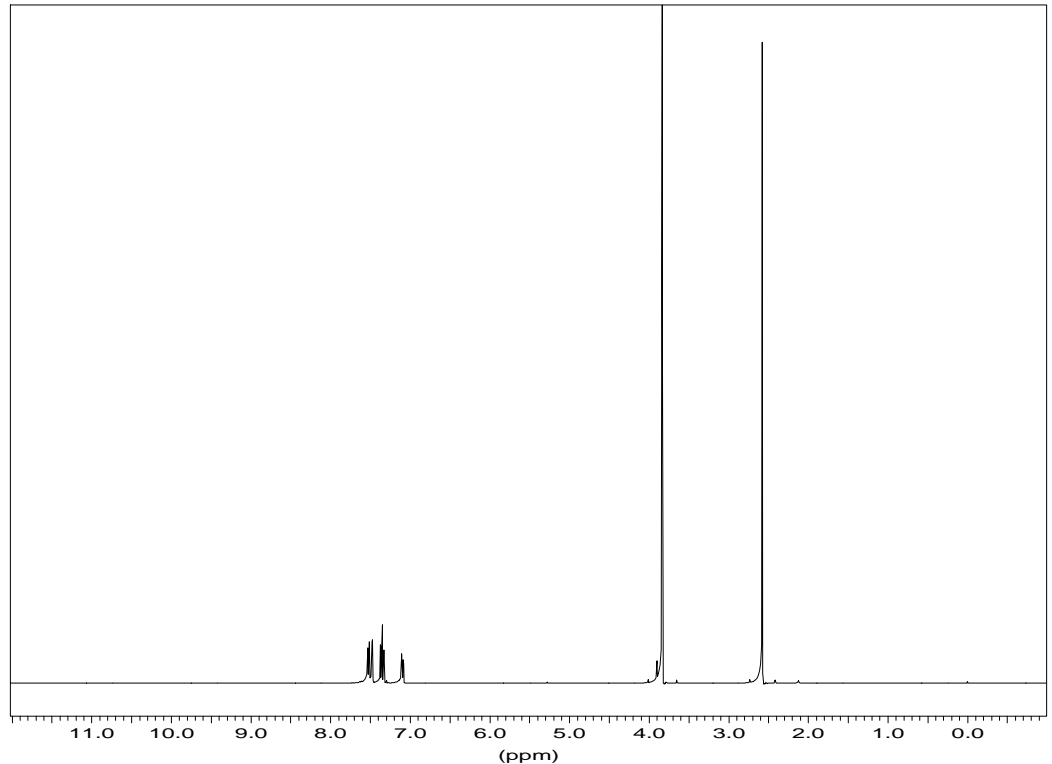

${ }^{13}$ C NMR

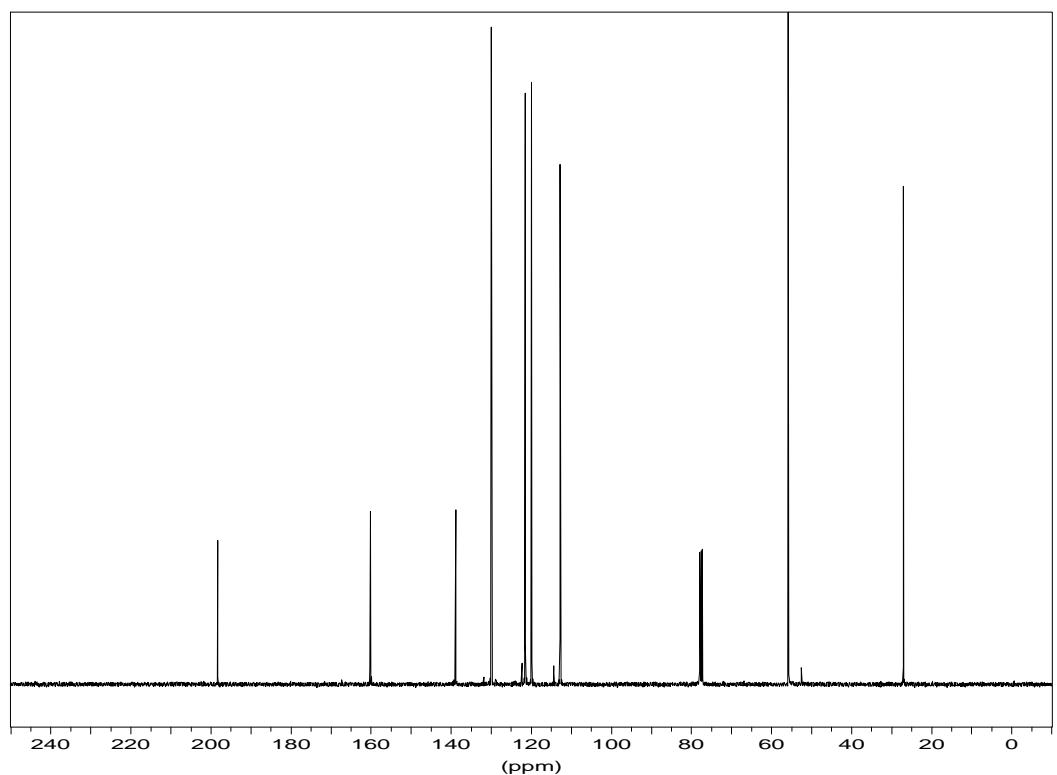




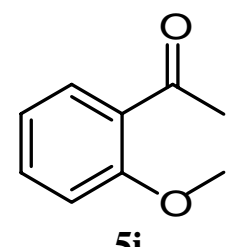

$5 i$

${ }^{1}$ HNMR

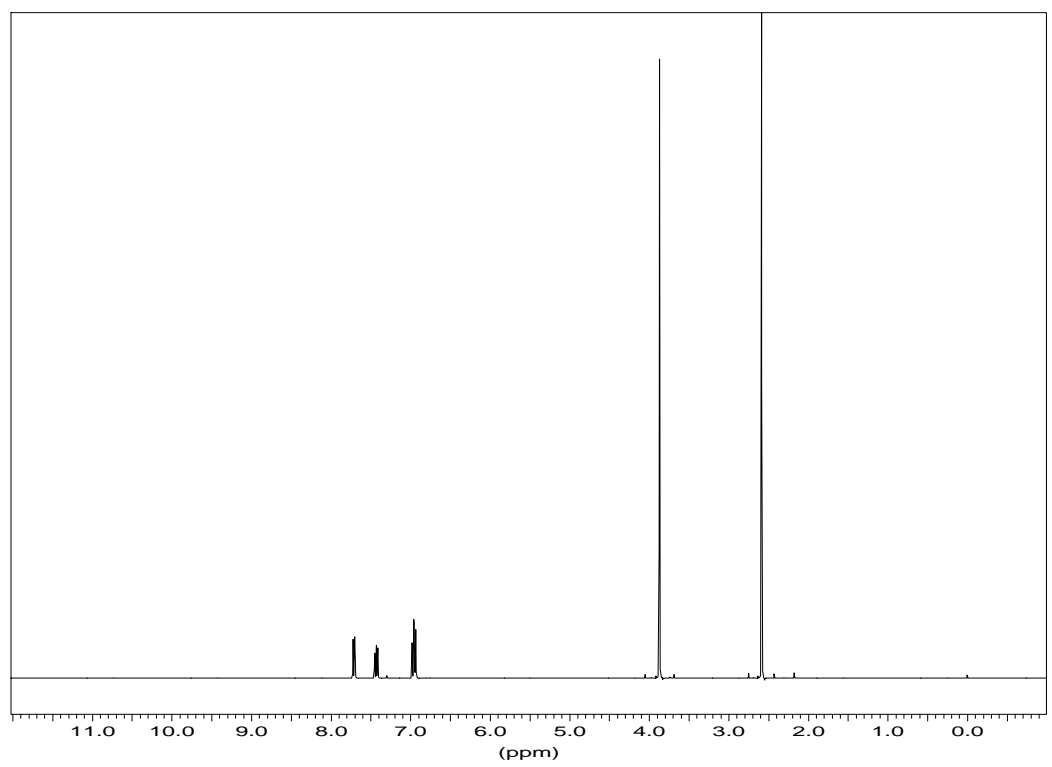

${ }^{13}$ C NMR

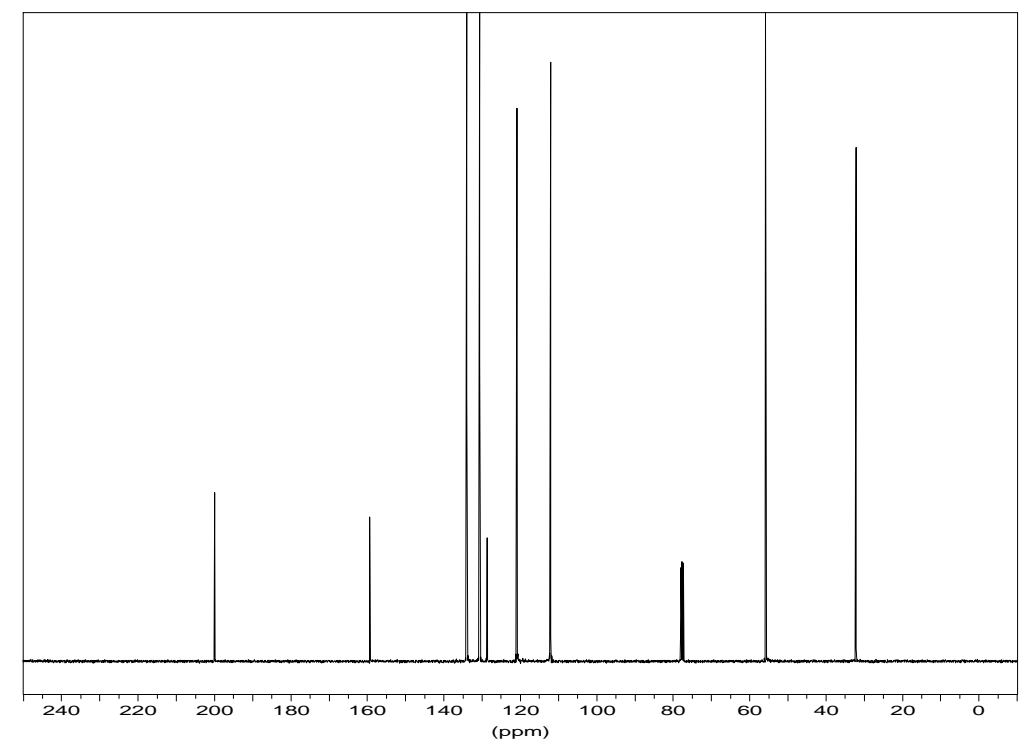


<smiles>CC(=O)c1ccc(C#N)cc1</smiles>

5j

${ }^{1} \mathrm{H}$ NMR

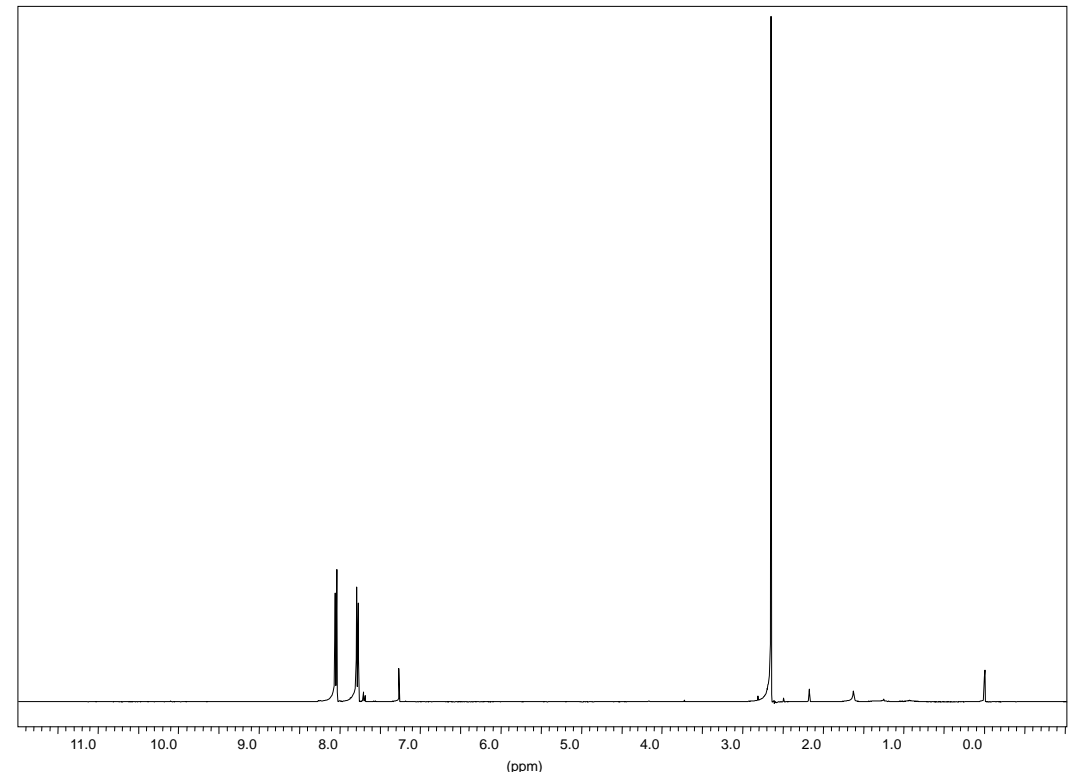

${ }^{13}$ C NMR

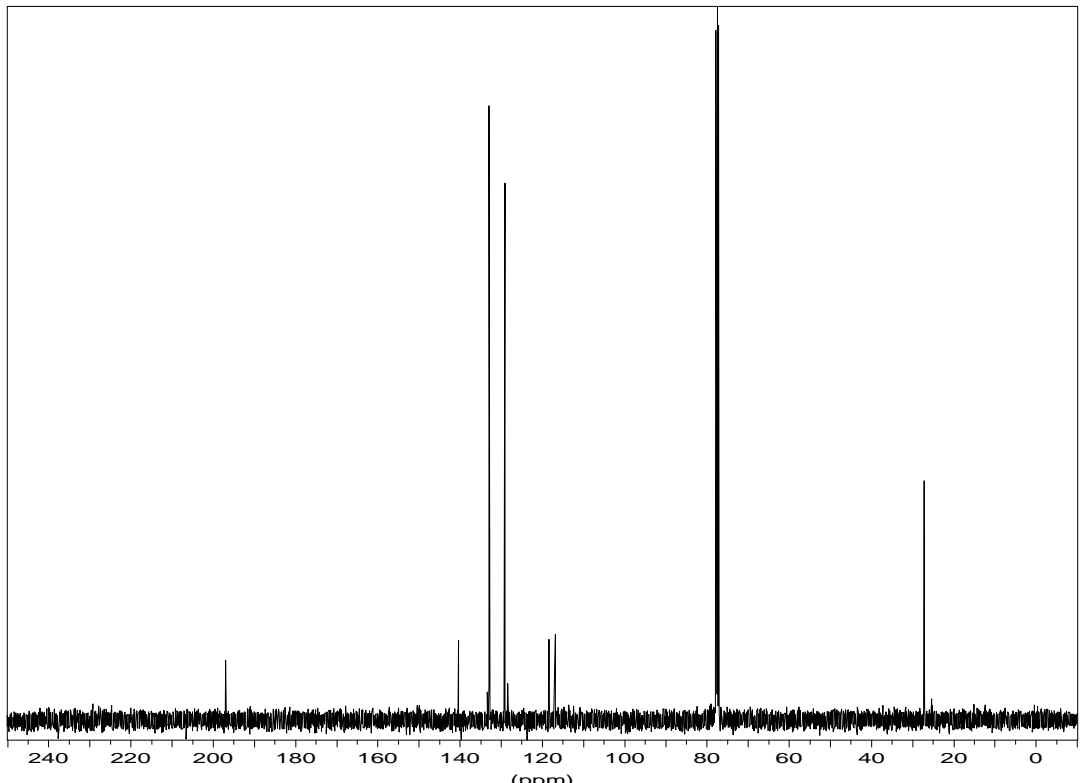




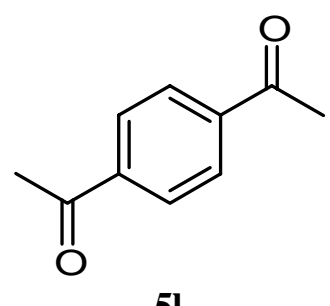

5l

${ }^{1}$ H NMR

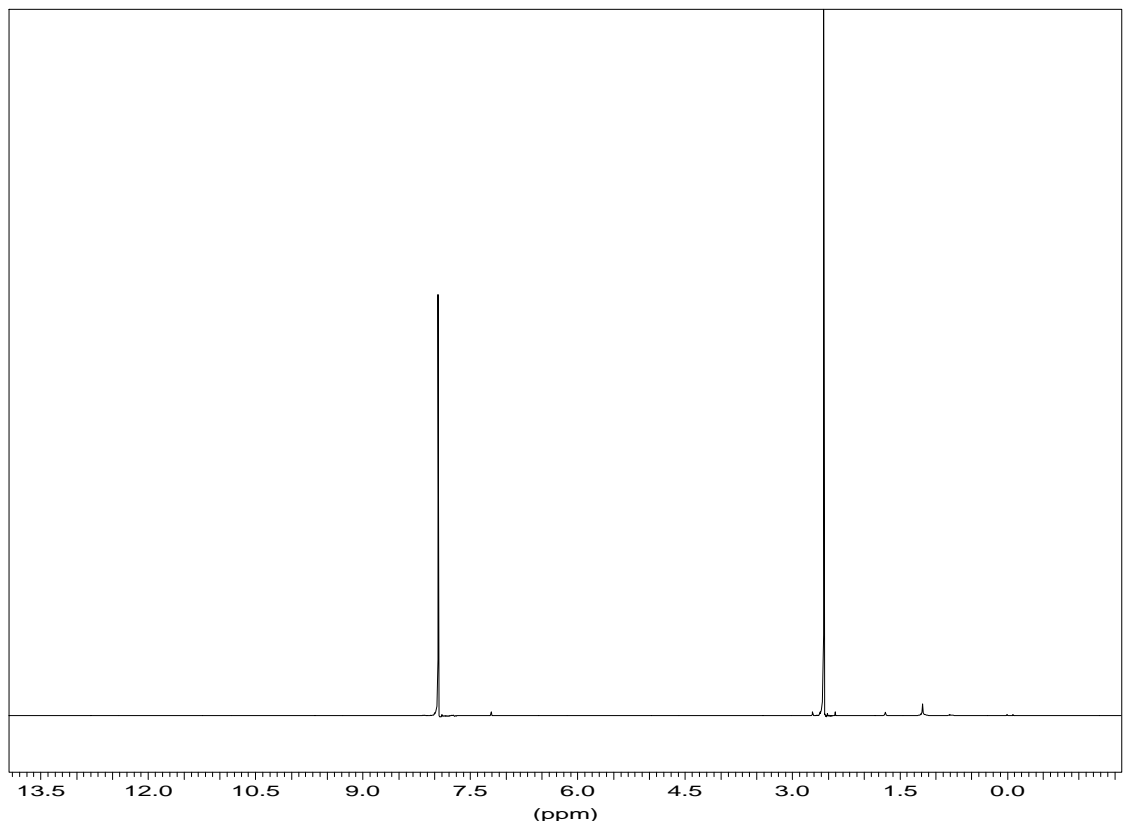

${ }^{13}$ C NMR

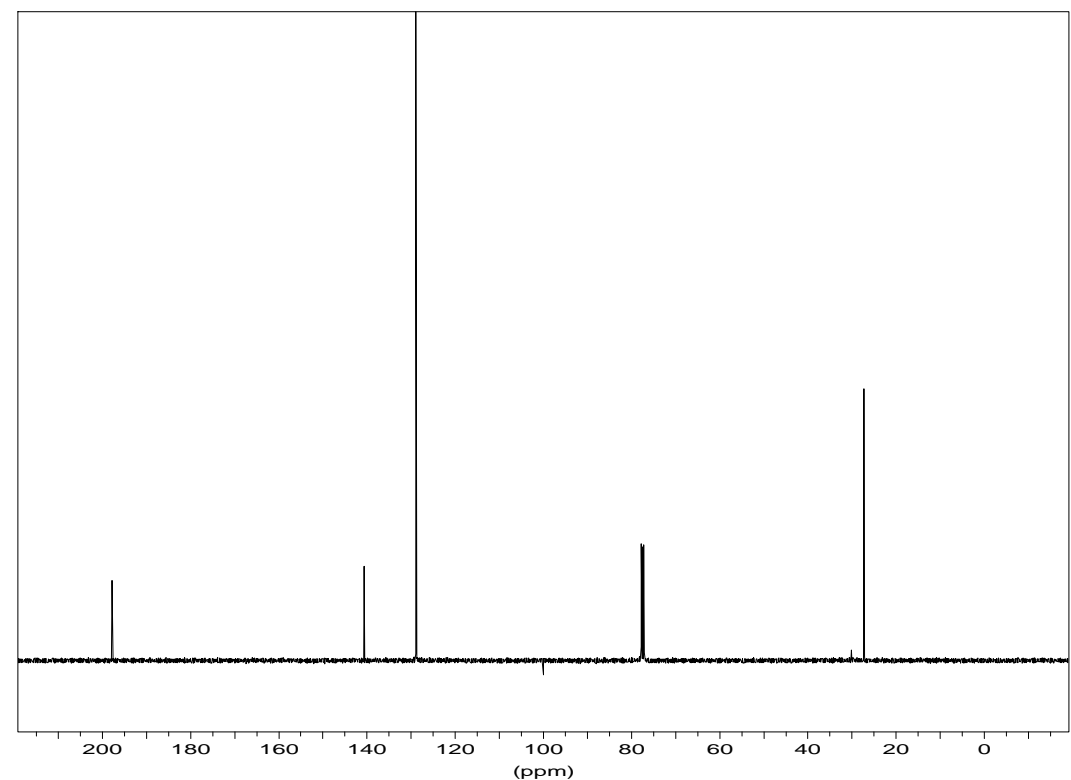




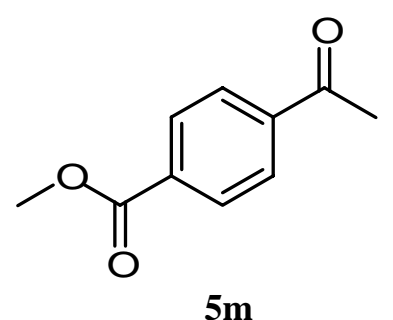

${ }^{1}$ H NMR

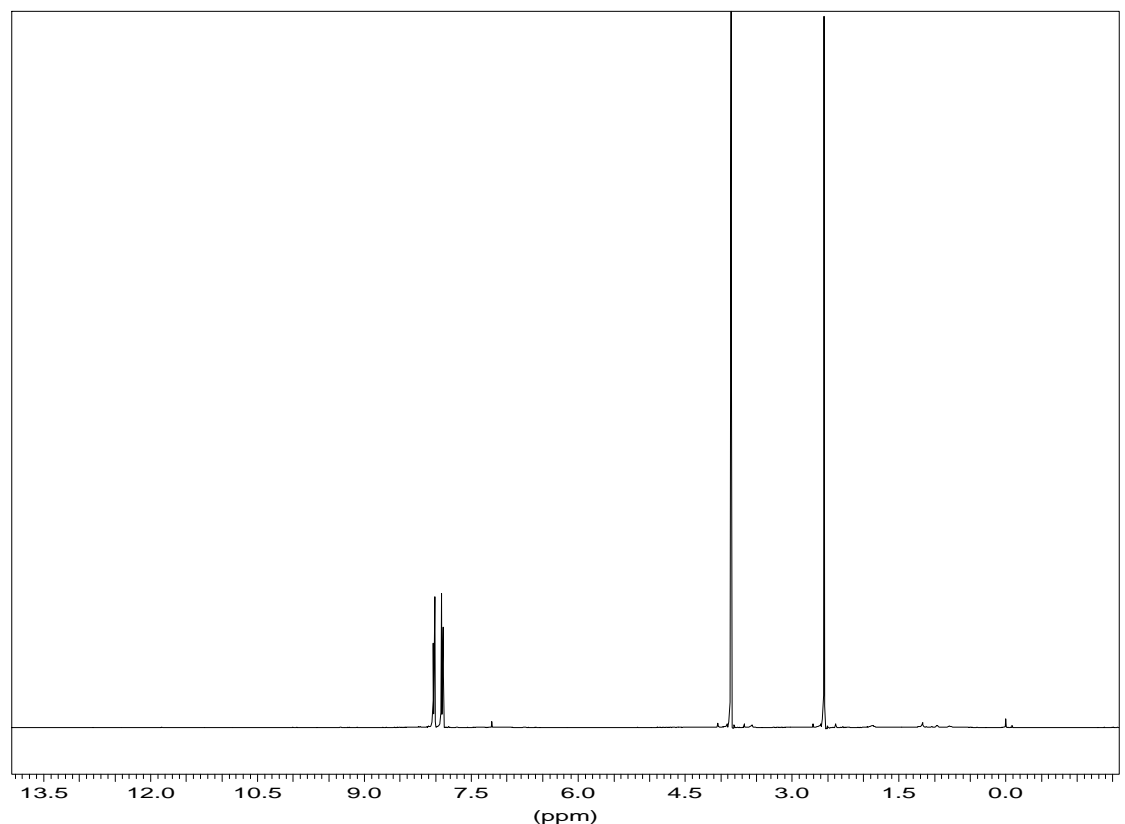

${ }^{13}$ C NMR

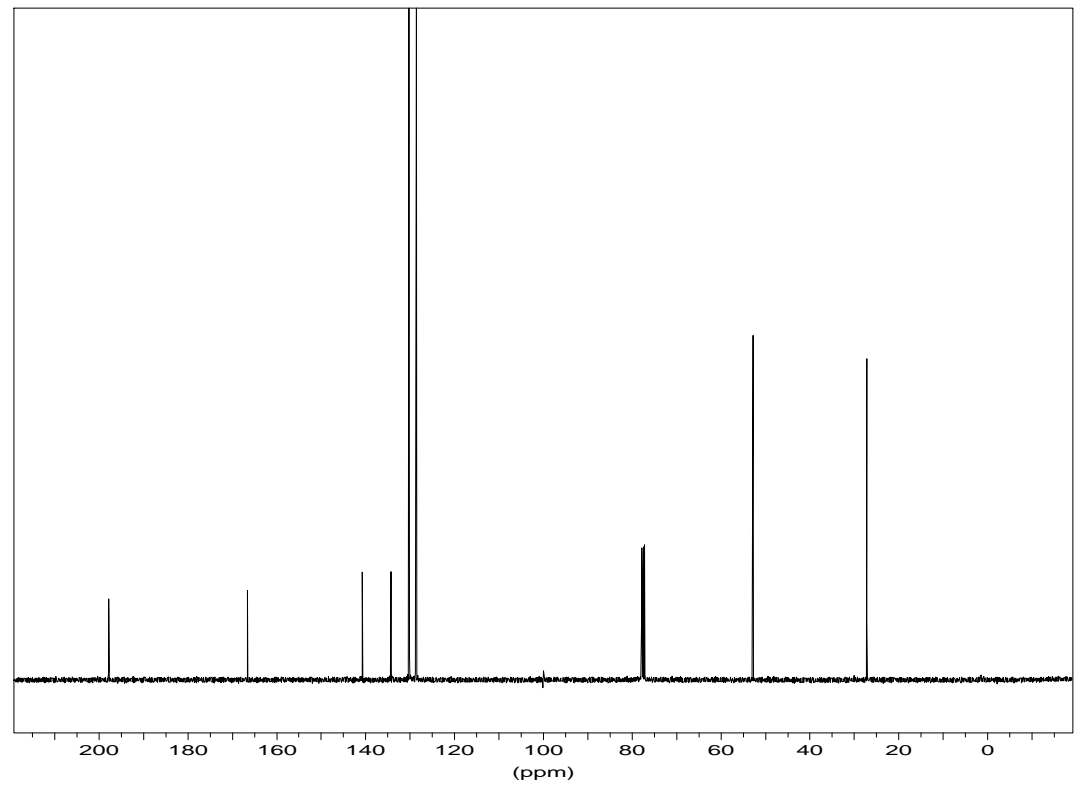

